\title{
Eficacia de una estrategia analgésica preincisional con ibuprofeno, paracetamol y dexametasona en colecistectomía laparoscópica. Estudio observacional prospectivo
}

\section{Efficacy of a preincisional analgesic strategy with ibuprofen, paracetamol and dexamethasone in laparoscopic cholecystectomy. Prospective observational study}

\author{
M. A. Pérez Herrero', S. López Álvarez², S. Galindo Menéndez³ y A. López García ${ }^{3}$
}

${ }^{1}$ Servicio de Anestesiología y Reanimación, Hospital Clínico Universitario de Valladolid, Valladolid, España.

2Servicio de Anestesiología y Reanimación, Hospital Abente y Lago, A Coruña, España. ${ }^{3}$ Servicio de

Anestesiología y Reanimación, Grupo HAM Hospitales, Madrid, España

\section{ABSTRACT}

Aims: To evaluate efficacy and security with a multimodal preventive analgesic technique (intravenous paracetamol, ibuprofen, dexametasone and local anesthetic infiltration incision] in postoperative pain management after laparoscopic cholecystectomy.

Methodology: Observational prospective study in laparoscopic cholecystectomy under common anesthetic protocol and multimodal pre-incisional analgesia using intravenous ibuprofen (800 mg), paracetamol ( $1 \mathrm{~g}$ ), dexamethasone $(0.1 \mathrm{mg} / \mathrm{kg}$ ) and $0.5 \%$ bupivacaine portals infiltration. Analgesic rescue with metamizole ( $2 \mathrm{~g}$ ) and morphine clorure (1 mg boluses). We noted pain grade, rescue analgesia, anesthetic-chirurgic complications and satisfaction level at five moments: in UCPA stay ( 20 min and $2 \mathrm{~h}$ after surgery and before discharge) and $24 \mathrm{~h}$ after surgery.

Results: 112 patients: 71 women and 41 men, $61.15 \pm 16.23$ aged; $76.20 \pm 12.68 \mathrm{~kg}$ weight, surgery time of $92.11 \pm 30.64$ minutes; $1.91 \pm 15,78$ microgrames $/ \mathrm{kg} / \mathrm{h}$ fentanyl doses; ASA I (34,8\%), II [40,2 \%), III (22,3 \%) y IV [2,7 \%). Two maximum pain peaks: at $20 \mathrm{~min}$ and at $24 \mathrm{~h}$ after the intervention and during movement. Severe pain and additional analgesia in 23 cases, and need for morphine chloride boluses

Pérez Herrero MA, López Álvarez S, Galindo Menéndez S, López García A. Eficacia de una estrategia analgésica preincisional con ibuprofeno, paracetamol y dexametasona en colecistectomía laparoscópica. Estudio observacional prospectivo. Rev Soc Esp Dolor 2020;27(2):104-112

\section{RESUMEN}

Objetivos: Evaluar la eficacia y seguridad de una técnica analgésica preventiva multimodal utilizando dexametasona, ibuprofeno y paracetamol preincisionales como estrategia analgésica perioperatoria tras colecistectomía laparoscópica.

Metodología: Estudio observacional prospectivo en pacientes sometidos a colecistectomía laparoscópica bajo un protocolo anestésico común y analgesia preincisional multimodal con ibuprofeno $(800 \mathrm{mg}$ ), paracetamol ( $1 \mathrm{~g}$ ) y dexametasona $[0,1 \mathrm{mg} / \mathrm{kg}$ ) intravenosos e infiltración de portales de inserción de trócares con bupivacaína 0,5\%; medicación analgésica intravenosa de rescate con metamizol (2 g) y cloruro mórfico (bolus de $1 \mathrm{mg}$ ). Se registraron intensidades de dolor mediante escala verbal numérica y escala categórica en reposo y movimiento, necesidad y dosis de analgesia de rescate, complicaciones anestésico-quirúrgicas y grado de satisfacción en Unidad de Recuperación Postanestésica (a los 20 min, 2 h de su ingreso y al alta) y a las $24 \mathrm{~h}$ de la intervención.

Resultados: Se analizaron datos de 112 pacientes: 71 mujeres y 41 hombres, con una media de edad de 61,15 $\pm 16,23$ años; un peso medio de 76,20 $\pm 12,68 \mathrm{~kg}$; duración del tiempo quirúrgico lentre

Recibido: 04-11-2019

Aceptado: 28-01-2020

Correspondencia: María A. Pérez Herrero mapeherrero@gmail.com 
in 6. Thirty cases complications were documented: emesis in 17 people (15.18\%), venous irritability in 10 cases $(8.93 \%$ ) and hemodynamic instability in 3 cases [2.68\%].

Conclusions: The multimodal analgesic strategy used in this study provided effective analgesia with fast, highquality anesthetic-surgical recovery in most patients. In case it was need, low perioperative opioid requirements. Low rate and severity of perioperative complications, which facilitated high outpatient surgery rates.

Key words: Multimodal analgesia, postoperative pain, laparoscopic cholecystectomy. incisión cutánea y cierre de portales de inserción de trócares fue de 92,11 \pm 30,64 min); se utilizaron dosis intraoperatorias de 1,91 $\pm 15,78 \mathrm{mcgs} / \mathrm{kg} / \mathrm{h}$ de fentanilo; el estado físico se calificó como de ASA I (34,8\%), II (40,2\%), III [22,3\%) y IV (2,7\%). Se objetivaron 2 picos máximos de dolor: a los 20 min y a las $24 \mathrm{~h}$ de intervención, más intenso en movimiento (inspiración forzada). En 23 casos se registró la presencia de dolor agudo intenso que requirió analgesia adicional (necesidad de cloruro mórfico en 6 casos). Se documentaron 30 casos de complicaciones perioperatorias: 17 (15,18\%) pacientes refirieron náuseas y/o vómitos, 10 (8,93\%) irritabilidad venosa y 3 [2,68\%) inestabilidad hemodinámica.

Conclusiones: La estrategia analgésica multimodal utilizada en este estudio proporcionó una analgesia eficaz con recuperación anestésico-quirúrgica rápida y de alta calidad en la mayoría de los pacientes, con bajos requerimientos de opioides perioperatorios, en los casos en los que se precisó. Baja incidencia y gravedad de complicaciones perioperatorias, lo que facilitó tasas elevadas de cirugía ambulatoria.

Palabras clave: Analgesia multimodal, dolor postoperatorio, colecistectomía laparoscópica.

\section{INTRODUCCIÓN}

Los avances quirúrgico-anestésicos y de gestión han posibilitado que la asistencia ambulatoria constituya el núcleo de la actividad asistencial. La colecistectomía laparoscópica en régimen ambulatorio ha demostrado resultados similares a la cirugía con ingreso (1). Las causas de hospitalización más frecuentes son el dolor mal controlado con analgésicos orales y las náuseas y/o vómitos postoperatorios, aunque también se han descrito otras complicaciones [?].

El dolor agudo postoperatorio es un problema más frecuente e intenso de lo esperado $[3,4)$. La analgesia inadecuada retrasa la recuperación integral del paciente (4), provoca insatisfacción (5), mayor estancia hospitalaria, reingresos, dolor crónico postquirúrgico y aumento de costes. En cambio, la analgesia eficaz disminuye la morbilidad, facilita el alta hospitalaria y la ambulatorización del procedimiento $[5,6]$.

El dolor postoperatorio asociado a colecistectomía laparoscópica es debido al traumatismo quirúrgico y al neumoperitoneo. La intensidad máxima se objetiva en las primeras $24 \mathrm{~h}$, para disminuir progresivamente [7]. Actualmente, existe consenso para utilizar estrategias analgésicas multimodales, preventivas y ahorradoras de opioides (8-10). Sin embargo, todavía no se dispone de pautas, protocolos y guías de administración de fármacos y medidas no farmacológicas perioperatorias, por lo que se hacen necesarios estudios para conocer y adoptar las mejores prácticas.

El objetivo de este estudio fue evaluar la eficacia de una estrategia analgésica multimodal, preincisional con paracetamol, ibuprofeno y dexametasona, asociada a la infiltración de los portales de inserción de trócares con anestésico local al finalizar la cirugía. Se registraron los síntomas asociados a la toma de ibuprofeno (náuseas y vómitos, dolor en el área de punción, irritabilidad vascular, etc.] como objetivo secundario.

\section{MATERIAL Y MÉTODOS}

Estudio observacional prospectivo aprobado por el Comité de Ética del Hospital Clínico de Valladolid (Presidente Dr. Pérez Rubio) con código PI 18-893, en junio de 2018 , en los pacientes intervenidos de colecistectomía laparoscópica entre el 1 de julio y el 31 de diciembre del año 2018, que aceptaron y firmaron el consentimiento informado para participar en el estudio.

Los criterios de inclusión fueron: pacientes intervenidos de colecistectomía laparoscópica con edad de 18-90 años, estado físico ASA I-III, con capacidad para entender y evaluar el dolor mediante las escalas empleadas [escala verbal numérica [EVN] o visual analógica [EVA] y categórica] y solicitar analgesia adicional. Se excluyeron pacientes que no cumplian los criterios de inclusión, con historia de tratamiento habitual domiciliario con opiáceos o alguno de los fármacos incluidos en el estudio, o con alergias o contraindicación para su utilización.

El procedimiento anestésico fue común en todos los casos. Tras una monitorización con electrocardiograma, presión arterial, pulsioximetría y profundidad anestésica [Sedline ${ }^{\circledR}$ Root $^{\circledR}$ Masimo ${ }^{\circledR}$, Miami, EE. UU.), se 
administraba ibuprofeno $800 \mathrm{mg}$, paracetamol $1 \mathrm{~g}$ y dexametasona $0,1 \mathrm{mg} / \mathrm{kg}$ de peso intravenosos; inducción intravenosa con propofol $(2 \mathrm{mg} / \mathrm{kg}$ ), y relajación muscular con rocuronio $[0,6 \mathrm{mg} / \mathrm{kg}]$. La vía aérea se preservaba mediante mascarilla laríngea AuraGain ${ }^{\circledR}$ del tamaño adaptado al peso. El mantenimiento fue realizado con sevoflurano para mantener niveles del Patient State Index (PSI) entre 30 y 40. La técnica quirúrgica se realizó bajo presión máxima de $12 \mathrm{~mm} \mathrm{Hg}$ de neumoperitoneo. En caso de objetivar una elevación de la presión arterial sistólica o de la frecuencia cardiaca mayor del $20 \%$ respecto a la basal se administraban bolus de fentanilo 100 mcgs, cada 5 min hasta recuperar la estabilidad hemodinámica. Al acabar la intervención se infiltraron los portales de entrada de trócares con bupivacaína 0,5\% con adrenalina $(10 \mathrm{ml})$.

Finalizada la intervención, los pacientes eran ingresados en la Unidad de Recuperación Postanestésica (URPA), donde se evaluó la intensidad de dolor a los 20 min y a las $2 \mathrm{~h}$ de su ingreso mediante escalas de dolor: EVA (el valor O se correspondía con ausencia total de dolor y 10 el peor dolor imaginable), y categórica con 5 categorías (sin dolor, dolor leve, moderado, intenso o insoportable), tanto en reposo como en movimiento (tos o inspiración profunda).

En caso de que el paciente refiriera un dolor moderado o intenso (EVA/EVN > 5 o dolor moderado-intenso con escala categórica) se administró analgesia adicional con metamizol intravenoso ( $2 \mathrm{~g}$ ), y en caso de persistencia del dolor se suministró analgesia de rescate con cloruro mórfico intravenoso en bolos de $1 \mathrm{mg} / 5 \mathrm{~min}$ hasta lograr analgesia satisfactoria (límite máximo de $10 \mathrm{mg}$ en $1 \mathrm{~h}$ y $30 \mathrm{mg}$ en $4 \mathrm{~h}$ ).

En todos los casos, se suministró una segunda dosis intravenosa de $800 \mathrm{mg}$ de ibuprofeno, antes del alta hospitalaria, $6 \mathrm{~h}$ después de la administración anterior. Posteriormente, la analgesia se aplicó por vía oral con $600 \mathrm{mg}$ de ibuprofeno y $1 \mathrm{~g}$ de paracetamol/8 $\mathrm{h}$, y en caso necesario, $100 \mathrm{mg}$ de tramadol como rescate. A las 24 h de la intervención se volvió a evaluar el dolor mediante EVN (O se correspondía con ausencia total de dolor y 10 el peor dolor imaginable) y categórica, tanto en reposo como en movimiento.

Se recogieron datos de intensidad de dolor, tomando como variables principales la diferencia entre los valores de EVA y escala categórica en reposo y movimiento respecto a valores basales en diferentes momentos del periodo postoperatorio en URPA la los $15 \mathrm{~min}$ de su ingreso y a las 2 h) y entre los valores de la EVN al día siguiente (24 h postintervención) por encuesta telefónica respecto a los valores basales y obtenidos en URPA; y la necesidad o no de rescate analgésico (metamizol, número de bolos y dosis total de cloruro mórfico intravenoso y de tramadol oral en domicilio). Se registraron los eventos adversos anestésico-quirúrgicos (hipotensión, náuseas y vómitos, sedación, eritema, dolor o signos de irritación venosa, que eran identificados y calificados por los investigadores como de gravedad entre 1 y 3 según escala de Likert $(0$ inexistente, 1 leve, 2 moderado, 3 grave y 4 muy grave).

La evaluación del dolor siempre fue realizada por enfermeras o anestesiólogos entrenados en su valoración, bien en la unidad de hospitalización, bien mediante llamada telefónica en caso de cirugía ambulatoria.
Se anotaron los siguientes datos: duración de la cirugía (considerando como momento cero la incisión, y tiempo de finalización el momento del cierre cutáneo); dosis total de fentanilo en microgramos considerando dosis bajas entre $0,5-2 \mathrm{mcg} / \mathrm{kg} / \mathrm{h}$ de intervención, moderadas entre $2-15 \mathrm{mcg} / \mathrm{kg} / \mathrm{h}$ y altas de $20-50 \mathrm{mcg} / \mathrm{kg} / \mathrm{h}$.

\section{Análisis estadístico}

El cálculo del tamaño muestral se realizó en base a los estudios previos de dolor postoperatorio (11). Los datos se introdujeron en una base de datos Excel $^{\circledR}$ (Microsoft Office, Microsoft, EE. UU.) y se analizaron mediante el paquete estadístico PASW ${ }^{\circledR}$ v22.0 (SPSS, Inc., Chicago, IL, EE. UU.).

Las variables cuantitativas se presentan con la mediana y la amplitud intercuartil, y las cualitativas según su distribución de frecuencias. Se utilizó el test de Kolmogorov Smirnov para comprobar la normalidad. Las comparaciones de las mediciones del dolor en reposo y en movimiento se realizaron mediante la prueba de Wilcoxon. Se calculó la significación asintótica bilateral entre las variables medidas. Aquellos valores de $p<0,05$ se consideraron estadísticamente significativos.

\section{RESULTADOS}

Se analizaron datos de 112 pacientes, tras excluir a 11 por diversos motivos resumidos en la Figura 1.

La Tabla I muestra datos demográficos, duración de la cirugía, consumo de fentanilo, estado físico y régimen en que se realizó el procedimiento en los 112 pacientes estudiados.

La Tablas II, III y IV indican la intensidad del dolor en diferentes momentos del estudio. Más de un $10 \%$ de pacientes presentaron dolor basal en reposo, porcentaje aún mayor con movimiento. Se encontraron importantes diferencias entre dolor en reposo y en movimiento a los 20 min de llegada a la URPA $(13,4$ vs. $26,79 \%$ según EVA y 26,78 vs. 33,92 \% en escala categórica) y a las $24 \mathrm{~h}$ de la intervención (ningún paciente dolor $>3$ según EVN frente a un porcentaje del $12,5 \%$ [reposo] y 19,61 \% [dinámico] según escala categórica].

Referente al consumo de fármacos, el 26,79\% de los pacientes precisaron analgesia adicional a los 20 min de llegada a la URPA. Tras administrar metamizol intravenoso, el alivio del dolor fue insuficiente en un $5,4 \%$ ( 6 pacientes) que precisaron rescate con cloruro mórfico ( 2 bolos de $1 \mathrm{mg}$ en 3 pacientes [2,7\%]) y 3 en otros 3 pacientes $(2,7 \%$ ). El consumo total de mórfico fue de 2,5 $\mathrm{mg}$ de media. Ningún paciente necesitó rescate con tramadol.

El 90,18\% de los procedimientos fueron ambulatorios, presentando buen control del dolor a las $2 \mathrm{~h}$ del ingreso en URPA. El tiempo de estancia en URPA fue de una media de $4,6 \mathrm{~h}$ con un mínimo de 3,5 y un máximo de 7. Los 10 pacientes ingresados presentaron una estancia media de 2,2 días (máximo de 5 y mínimo de 1 día). En su domicilio no se registraron valores 


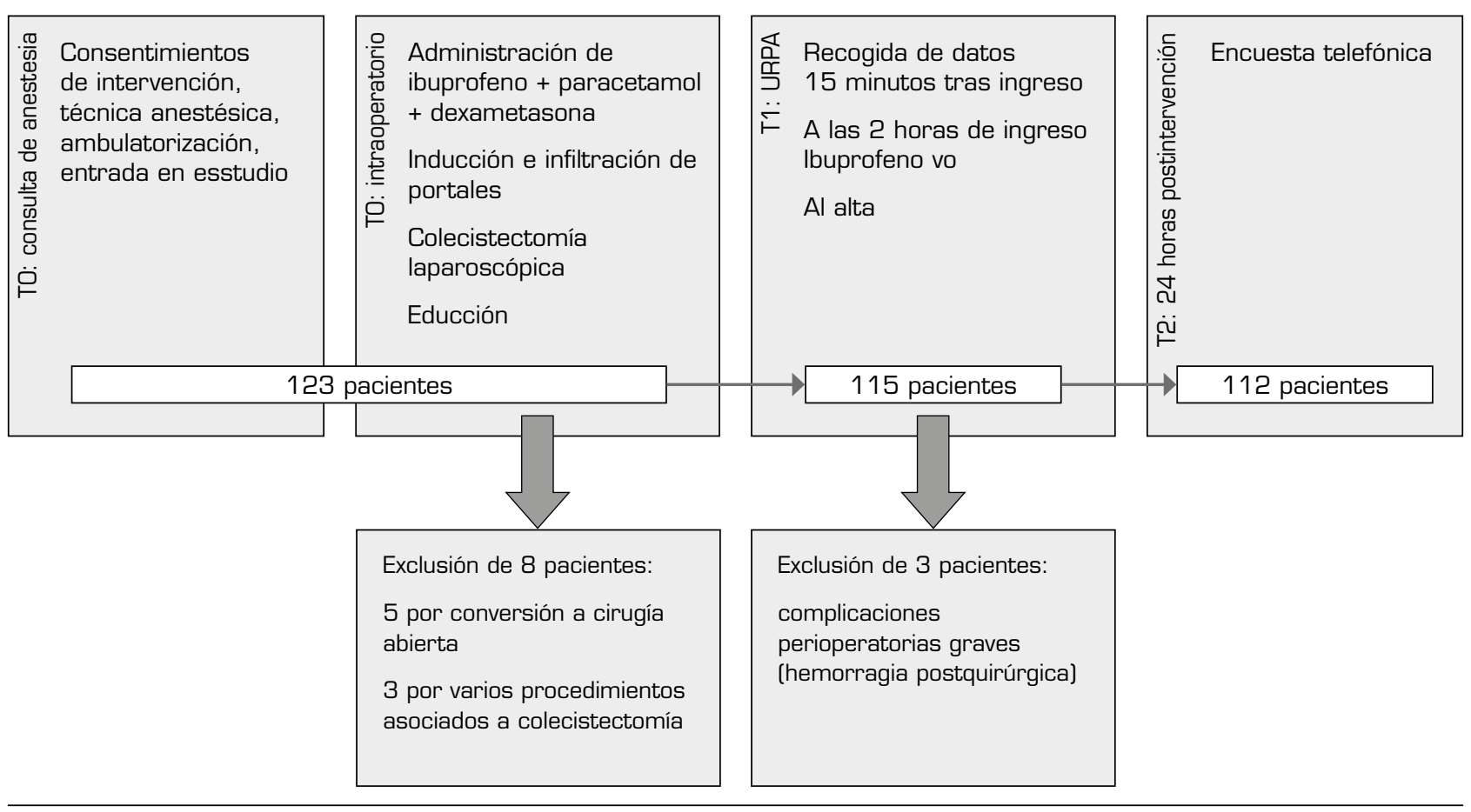

Fig. 1. Resumen del protocolo de estudio. TO es el momento de solicitar el consentimiento informado para el estudio, en la consulta de preanestesia. T1: a los 15 min de su ingreso en la Unidad de Recuperación Postanestésica (URPA) se recogen datos de intensidad de dolor mediante escala categórica y EVA. Caso de EVA o EVN (encuesta telefónica) > 3 (o escala categórica de dolor moderado-intenso) se administraba analgesia de rescate con metamizol [ 2 g] intravenoso. A 30 min de su administración, y caso de persistencia del dolor, cloruro mórfico en bolus de 1 mg hasta conseguir analgesia satisfactoria. Se anotaron datos de las siguientes variables:

- Datos de filiación: edad, sexo, peso.

- Intraoperatorio: dosis de fentanilo intravenoso, duración de la cirugía, complicaciones.

- Postoperatorio:

I En URPA (en 3 momentos: a los 20 min, a las 2 h del ingreso y al alta): intensidad de dolor (puntuación de EVA y escala categórica al ingreso, dosis total de analgesia de rescate), complicaciones, satisfacción del anestesiólogo responsable con la técnica.

I 24 h tras intervención: intensidad de dolor (puntuación EVA y escala categórica, dosis de tramadol), complicaciones, satisfacción del paciente.

TABLA I

DATOS DEMOGRÁFICOS (SEXO, EDAD, PESO Y ESTADO FÍSICO), DURACIÓN DE LA CIRUGÍA, CONSUMO TOTAL FENTANILO INTRAOPERATORIO, RÉGIMEN EN QUE SE REALIZÓ EL PROCEDIMIENTO (HOSPITALIZACIÓN O AMBULATORIO) Y CIRUGÍA URGENTE O PROGRAMADA

\begin{tabular}{|l|l|}
\hline Sexo & 71 mujeres $[63,4 \%] / 41$ hombres $[36,6 \%]$ \\
\hline Años & $61,15 \pm 16,22$ \\
\hline Peso (kg) & $76,20 \pm 12,68$ \\
\hline Estado físico ASA I/II/III/IV & $39 / 45 / 25 / 3$ \\
\hline Duración de la cirugía (min) & $92,11 \pm 30,64$ \\
\hline Fentanilo intraoperatorio & $3,46 \pm 1,75 \mathrm{mcg} / \mathrm{kg} / \mathrm{h}(1,5-4,95)$ \\
\hline Régimen ambulatorio/ingreso & $223,66 \pm 102,22(100-600)$ por paciente \\
\hline Cirugía urgente/programada & $101(90,18 \%] / 11(9,22 \%)$ \\
\hline
\end{tabular}

Los valores se expresan como número y porcentaje, o media y desviación estándar. En el caso del fentanilo intraoperatorio se reflejan entre paréntesis los valores máximo y mínimo. 
TABLA ॥

RESULTADOS DE INTENSIDAD DE DOLOR MEDIDOS POR ESCALA EVA EN REPOSO Y MOVIMIENTO EN 3 MOMENTOS: BASAL, URPA Y A LAS 24 H DE LA INTERVENCIÓN

\begin{tabular}{|c|c|c|c|c|}
\hline & EVA reposo & Porcentaje & EVA dinámico & Porcentaje \\
\hline \multirow{4}{*}{ Basal } & \multirow{2}{*}{$0-3$} & \multirow{2}{*}{88,5} & $0-3$ & 52,9 \\
\hline & & & $4-6$ & 35,6 \\
\hline & \multirow{2}{*}{ 4-6 } & \multirow{2}{*}{11,5} & $0-3$ & 0 \\
\hline & & & $4-6$ & 11,5 \\
\hline \multirow{4}{*}{ URPA (20 min del ingreso) } & \multirow{2}{*}{$0-3$} & \multirow{2}{*}{79,47} & $0-3$ & 73,2 \\
\hline & & & $4-6$ & 6,27 \\
\hline & \multirow{2}{*}{$4-6$} & \multirow{2}{*}{20,53} & $0-3$ & 0 \\
\hline & & & $4-6$ & 20,53 \\
\hline \multirow{4}{*}{ URPA (2 h del ingreso) } & \multirow{2}{*}{$0-3$} & \multirow{2}{*}{99,9} & $0-3$ & 38,4 \\
\hline & & & 4-6 & 61,5 \\
\hline & \multirow{2}{*}{$4-6$} & \multirow{2}{*}{0} & $0-3$ & 0 \\
\hline & & & $4-6$ & 0 \\
\hline \multirow{4}{*}{ Postoperatorio inmediato ( 24 h tras intervención) } & \multirow{2}{*}{$0-3$} & \multirow{2}{*}{94,7} & $0-3$ & 51 \\
\hline & & & $4-6$ & 43,7 \\
\hline & \multirow{2}{*}{ 4-6 } & \multirow{2}{*}{5,3} & $0-3$ & 0 \\
\hline & & & 4-6 & 5,3 \\
\hline
\end{tabular}

EVA: escala visual analógica. URPA: Unidad de Recuperación Postanestésica.

En negrita se resaltan los resultados más frecuentes en cada momento de medición.

TABLA III

RESULTADOS DE INTENSIDAD DE DOLOR MEDIDOS MEDIANTE ESCALA CATEGÓRICA DE 5 ÍTEMS (SIN DOLOR, LEVE, MODERADO, INTENSO E INSOPORTABLE) EN REPOSO Y MOVIMIENTO EN 3 MOMENTOS: BASAL, URPA Y A LAS 24 H DE LA INTERVENCIÓN

\begin{tabular}{|c|c|c|c|c|}
\hline & Categórica reposo & Porcentaje & Categórica movimiento & Porcentaje \\
\hline \multirow{4}{*}{ Basal } & \multirow{2}{*}{$\begin{array}{l}\text { Sin dolor (0) } \\
\text { o leve (1) }\end{array}$} & \multirow[b]{2}{*}{56,3} & Sin dolor (0) o leve (1) & 44,7 \\
\hline & & & $\begin{array}{l}\text { Moderado (2) o intenso } \\
\text { [3]-insoportable [4] }\end{array}$ & 11,6 \\
\hline & \multirow{2}{*}{$\begin{array}{l}\text { Moderado (2)/ } \\
\text { intenso (3) o } \\
\text { insoportable (4) }\end{array}$} & \multirow[b]{2}{*}{43,8} & Sin dolor (0) o leve [1] & 4,5 \\
\hline & & & $\begin{array}{l}\text { Moderado (2)-intenso } \\
\text { (3)-insoportable (4) }\end{array}$ & 39,3 \\
\hline \multirow{4}{*}{ URPA (20 min del ingreso] } & \multirow{2}{*}{$\begin{array}{l}\text { Sin dolor }[0] \\
\text { o leve (1) }\end{array}$} & \multirow[b]{2}{*}{75,89} & Sin dolor (0) o leve (1) & 60,71 \\
\hline & & & $\begin{array}{l}\text { Moderado (2) o intenso } \\
\text { (3)-insoportable (4) }\end{array}$ & 15,18 \\
\hline & \multirow{2}{*}{$\begin{array}{l}\text { Moderado (2) } \\
\text { o intenso (3) o } \\
\text { insoportable (4) }\end{array}$} & \multirow[b]{2}{*}{24,1} & Sin dolor (0) o leve [1] & 7,89 \\
\hline & & & $\begin{array}{l}\text { Moderado (2) o intenso } \\
\text { (3)-insoportable [4] }\end{array}$ & 16,21 \\
\hline \multirow{4}{*}{ URPA ( $2 \mathrm{~h}$ de ingreso] } & \multirow{2}{*}{$\begin{array}{l}\text { Sin dolor }[0] \\
\text { o leve (1] }\end{array}$} & \multirow[b]{2}{*}{76,8} & Sin dolor (0) o leve (1) & 52,7 \\
\hline & & & $\begin{array}{l}\text { Moderado (2)-intenso } \\
\text { (3)-insoportable (4) }\end{array}$ & 24,1 \\
\hline & \multirow{2}{*}{$\begin{array}{l}\text { Moderado [2], } \\
\text { intenso [3] o } \\
\text { insoportable }\end{array}$} & \multirow[b]{2}{*}{23,2} & Sin dolor (0) o leve (1) & 8,9 \\
\hline & & & $\begin{array}{l}\text { Moderado (2) o intenso } \\
\text { [3]-insoportable (4] }\end{array}$ & $14,3 \%$ \\
\hline
\end{tabular}


TABLA III [CONT.]

RESULTADOS DE INTENSIDAD DE DOLOR MEDIDOS MEDIANTE ESCALA CATEGÓRICA DE 5 ÍTEMS (SIN DOLOR, LEVE, MODERADO, INTENSO E INSOPORTABLE] EN REPOSO Y MOVIMIENTO EN 3 MOMENTOS: BASAL, URPA Y A LAS 24 H DE LA INTERVENCIÓN

\begin{tabular}{|c|c|c|c|c|}
\hline & Categórica reposo & Porcentaje & Categórica movimiento & Porcentaje \\
\hline \multirow{4}{*}{$\begin{array}{l}\text { Postoperatorio inmediato } \\
\text { (24 h tras intervención) }\end{array}$} & \multirow{2}{*}{$\begin{array}{l}\text { Sin dolor }(0) \\
\text { o leve }(1)\end{array}$} & \multirow[b]{2}{*}{87,6} & Sin dolor $(0)$ o leve $(1)$ & 75 \\
\hline & & & $\begin{array}{l}\text { Moderado (2), intenso (3) o } \\
\text { insoportable }\end{array}$ & 12,6 \\
\hline & \multirow{2}{*}{$\begin{array}{l}\text { Moderado (2), } \\
\text { intenso (3) o } \\
\text { insoportable }\end{array}$} & \multirow[b]{2}{*}{12,5} & Sin dolor (0) o leve (1) & 0 \\
\hline & & & $\begin{array}{l}\text { Moderado (2), intenso (3) o } \\
\text { insoportable }\end{array}$ & 12,5 \\
\hline
\end{tabular}

URPA: Unidad de Recuperación Postanestésica.

En negrita se resaltan los resultados más frecuentes en cada momento de medición.

TABLA IV

DIFERENCIA DE LA INTENSIDAD DE DOLOR CUANTIFICADO MEDIANTE ESCALA EVA Y CATEGÓRICA EN REPOSO Y MOVIMIENTO. SE REPRESENTAN LAS MEDIAS DE LA INTENSIDAD DE DOLOR EN 2 MOMENTOS DEL POSTOPERATORIO (ESTANCIA EN URPA Y 24 H TRAS INTERVENCIÓN) TANTO EN REPOSO COMO EN MOVIMIENTO

\begin{tabular}{|l|l|c|c|}
\hline & $\begin{array}{c}\text { Diferencia entre dolor } \\
\text { en URPA y basal }\end{array}$ & $\begin{array}{c}\text { Diferencia entre } \\
\text { dolor a las 24 } \mathrm{h} \text { de la } \\
\text { intervención y basal }\end{array}$ & $\begin{array}{c}\text { Diferencia entre } \\
\text { dolor a las 24 } \mathrm{h} \text { de la } \\
\text { intervención y en URPA }\end{array}$ \\
\hline EVA reposo & $-0,85 \pm 1,27$ (-5 a 1) & $-1,01 \pm 1,39$ (-5 a 2) & $-0,49 \pm 1,43$ (-5 a 5) \\
\hline EVA movimiento & $0,95 \pm 1,00$ (-6 a 3) & $-1,24 \pm 1,14(-3$ a 2) & $-0,54 \pm 1,32$ (-6 a 2) \\
\hline Escala categórica reposo & $-0,65 \pm 1,03$ (-3 a 1) & $-0,65 \pm 1,10$ (-3 a 1) & $0,13 \pm 0,99$ (-3 a 2) \\
\hline Escala categórica movimiento & $-0,75 \pm 1,38$ (-5 a 2) & $-0,58 \pm 1,14(-3$ a 2) & $0,01 \pm 1,15$ (-3 a 4) \\
\hline
\end{tabular}

EVA: escala visual analógica. URPA: Unidad de Recuperación Postanestésica.

Se expresan datos de la media y desviación estándar, indicando entre paréntesis los valores máximo y mínimo.

superiores a 3 en EVN, pero un 3,6 \% relataron dolor intenso en reposo y un 7,1 \% en movimiento, al aplicar la escala categórica.

Únicamente se hallaron diferencias significativas ( $p=0,002$ ) entre la intensidad medida mediante EVN en movimiento a las $24 \mathrm{~h}$ de la intervención respecto a la situación basal y la EVA en reposo en los mismos momentos.

Se registraron complicaciones en 30 pacientes: 17 casos $(15,18 \%)$ presentaron náuseas y/o vómitos, en 10 casos $[8,93 \%$ ) hubo dolor en el punto de infusión o irritabilidad venosa caracterizada por eritema y prurito (6 fueron calificados como leve y 4 como moderado) y en 3 casos $[2,68 \%$ ) inestabilidad hemodinámica.

Las causas de ingreso de los 10 pacientes estudiados fueron las siguientes: hallazgo intraoperatorio de colecistitis en 5 casos, complicaciones perioperatorias refractarias al tratamiento (hipotensión) en 3 casos y por negativa del paciente al alta domiciliaria en otros 2 casos.

La tasa de reingreso de pacientes fue del $0 \%$.

\section{DISCUSIÓN}

La colecistectomía laparoscópica es un procedimiento de mediana-baja complejidad, que se asocia con una morbilidad baja, por debajo del $2,2 \%$ (11), complicaciones poco frecuentes $(0,1-0,6 \%)$ y dolor de intensidad leve-moderada, lo que facilita su ambulatorización (12). El dolor postoperatorio es una respuesta adaptativa caracterizada por ser predecible, multifactorial y de corta duración, pero de intensidad mayor de la esperada y se exacerba con el movimiento.

La complejidad del dolor asociado a la colecistectomía laparoscópica ha propiciado numerosas investigaciones para optimizar su abordaje (13). Su valor máximo suele registrarse a los 20 min de ingreso en URPA, para descender progresivamente a los $60 \mathrm{~min}$, hasta desaparecer en $24 \mathrm{~h}$ en el $75,6 \%$ de los casos (14). Sin embargo, con la deambulación y los movimientos inspiratorios, el porcentaje de pacientes con dolor intenso es mayor del deseable, con las repercusiones que ello 
tiene sobre la satisfacción del paciente y la inclusión de la colecistectomía laparoscópica en programas de cirugía ambulatoria (15).

El dolor parietal es el dominante en esta intervención, y la eficacia de las estrategias analgésicas multimodales junto con la infiltración de los puertos de entrada de los trócares con anestésico local de larga duración se ha demostrado eficaz en el control del dolor en las primeras 4-8 h y a las $24 \mathrm{~h}$ postcirugía. El 7,1 \% de los pacientes referían dolor intenso en la escala categórica a las $24 \mathrm{~h}$ de la intervención en movimiento, que no se corresponde con los valores de EVA ni con la utilización de la medicación de rescate. Este dato puede explicarse por la persistencia de la sensibilización central a pesar de la desaparición del estímulo nociceptivo, pero también a falta de entrenamiento, de información-educación, o a la pauta analgésica domiciliaria insuficiente $[3,13,14)$.

En este estudio es llamativa la elevada intensidad de dolor basal registrada antes de la cirugía, que incluso supera a los valores registrados en el periodo postoperatorio inmediato. Este hecho se podría explicar por la alta proporción de pacientes intervenidos en fase de colecistitis aguda; con tratamiento analgésico deficiente. Esta deficiencia en el tratamiento de dolor agudo de origen biliar (colelitiasis o colecistitis aguda) se encuentra en varios ensayos clínicos multicéntricos recientes $(4,16,17)$. Otras explicaciones posibles a esta alta intensidad de dolor previo a la intervención serían las limitaciones propias de las escalas empleadas, o por sesgos de la interpretación, recogida o transcripción de los datos.

La estrategia analgésica utilizada en el estudio permitió minimizar la utilización de opioides en el periodo perioperatorio (dosis bajas-moderadas de fentanilo intraoperatorio). El porcentaje de paciente que precisó rescate con opioides es pequeño y las dosis utilizadas muy bajas.

El dolor postoperatorio es multifactorial, desconociendo la importancia que tienen ciertas características del paciente (edad, vulnerabilidad psicológica), así como aspectos relacionados con la cirugía (número y tamaño de incisiones, presión de neumoperitoneo, drenaje de gas, bilis o sangre intrabdominal y drenaje subhepático, bloqueos nerviosos, reclutamiento pulmonar, infusiones intraperitoneales) (17-25).

En nuestro estudio, el porcentaje de pacientes que fueron ambulatorizados es alto $(90,18 \%)$, y los resultados tanto de eficacia analgesia como de complicaciones (náuseas y vómitos] son mejores que en otros estudios realizados (14), a pesar de haber utilizado solo dexametasona como estrategia de profilaxis. El estudio de López-Torres incluye a pacientes ginecológicas (mayor riesgo de náuseas y vómitos) y no administraron dexametasona de forma sistemática, mientras que en nuestro estudio los pacientes presentaban un buen estado físico y las dosis de fentanilo y cloruro mórfico en la URPA utilizadas fueron bajas. En cambio, los resultados de eficacia eran similares a los descritos en otros estudios con ibuprofeno preincisional (6) a menores dosis u otras estrategias analgésicas multimodales [21-28].

Hay que destacar que en el estudio de Ahiskalioglu y cols. [6] consiguen resultados similares de analgesia a la obtenida en este estudio, con la utilización de una dosis de $400 \mathrm{mg}$ de ibuprofeno por vía oral o intravenosa, sin asociar otra medida farmacológica, previa a la incisión quirúrgica. Sin embargo, estos autores no realizan seguimiento al alta hospitalaria ni pautan tratamiento domiciliario.

Entre las pautas analgésicas eficaces se han descrito medidas farmacológicas (opioides, AINE $(10,20)$, paracetamol [29], gabapentinoides, dexametasona (10), lidocaína, sulfato de magnesio, ketamina y agonistas alfa-2 [30]) y no farmacológicas (infiltración de los portales de inserción de trócares con anestésico local, presión baja de neumoperitoneo, lavado con suero salino tras la intervención y aspiración de neumoperitoneo]. En este estudio seguimos las recomendaciones y tratamiento domiciliario asociando ibuprofeno y paracetamol por vía oral, siguiendo las pautas de la Cochrane (25). Desaconsejan anestésico local intraperitoneal y bloqueos del plano transverso abdominal, y técnicas quirúrgicas utilizando un único puerto.

La mayoría de los eventos adversos relacionados con la administración de ibuprofeno se asocian con el consumo crónico del mismo o en pacientes que presentan limitaciones para su utilización. La administración intravenosa debe realizarse lentamente (30 min), pero a pesar de ello se puede producir dolor en el lugar de punción y signos irritativos de la vena canalizada. En nuestro estudio se produjo en un 8,93\% de los pacientes, aunque en ningún caso fue de gravedad, consistiendo en eritema y dermografismo en la zona de punción autolimitada, que no obligó a modificar la velocidad de infusión del fármaco (31).

Sin embargo, este trabajo presenta importantes limitaciones. Se trata de un estudio observacional, sin comparar con ningún grupo control u otra técnica analgoanestésica, con los sesgos que conlleva; en un único centro, lo que limita la validez externa. Además, los resultados podrían infra o sobrestimarse, al realizarse únicamente en pacientes programados de colecistectomía laparoscópica bajo anestesia general, procedimiento que asocia baja intensidad y duración de dolor postoperatorio (31-34).

Otras limitaciones son la no inclusión de otras medidas analgésicas de eficacia demostrada. Solo se estudió la intensidad de dolor localizado en hipocondrio derecho, sin tener en cuenta otras localizaciones posibles (zona de punción venosa, orofaringe, espalda u hombro derecho]. Además, la utilización de escalas subjetivas de evaluación del dolor no cubre todas las dimensiones del dolor.

En conclusión, la estrategia analgésica multimodal utilizada en este estudio proporcionó una analgesia eficaz con recuperación anestésico-quirúrgica rápida y de alta calidad en la mayoría de los pacientes estudiados. En caso de precisar opioides perioperatorios, se utilizaron a dosis bajas. No se registraron síntomas graves asociados a la técnica analgésica, lo que facilitó tasas elevadas de cirugía ambulatoria.

\section{CONFLICTOS DE INTERESES}

Los autores declaran no tener ningún conflicto de intereses. 


\section{FUENTES DE FINANCIACIÓN}

Este estudio ha sido financiado parcialmente por Solibu ${ }^{\circledR}$ Altan Pharmaceuticals SAU.

\section{PARTICIPACIÓN DE LOS AUTORES}

Todos los autores han participado en el diseño, recogida de datos y redacción final del manuscrito.

\section{BIBLIOGRAFÍA}

1. Tang H, Dong A, Yan L. Day surgery versus overnight stay laparoscopic cholecystectomy: A systematic review and meta-analysis. Dig Liver Dis. 2015;47(7):556-6. DOI: 10.1016/j.dld.2015.04.007.

2. Díez Castillo E, Telletxea Benguria S, Intxaurraga Fernández K, Esnaola Iriarte B. Parálisis diafragmática unilateral tras colecistectomía laparoscópica. Rev Esp Anestesiol Reanim. 2019;66(6):342-5 DOI: 10.1016/j.redar.2019.01.006.

3. Aguilar JL. Situación actual del dolor agudo postoperatorio en el Sistema Nacional de Salud. Las tecnologías de la información y comunicación ayudan a conseguir un hospital (y atención primaria) sin dolor. Rev Soc Esp Dolor. 2019;26(3):13943. DOI: 10.20986/resed.2019.3739/2019.

4. Montes A, Aguilar JL, Benito MC, Caba F, Margarit C. Acute Pain Group of the Spanish Society (SED). Management of postoperative pain Spain: a nationwide survey of practice. Acta Anaesthesiol Scand. 2017;61(5):480-91. DOI: 10.1111/aas. 12876.

5. Pérez Herrero MA, López Álvarez S, Fadrique Fuentes A, Manzano Lorefice F, Bartolomé Bartolomé C, González de Zárate J. Quality of postoperative recovery after breast surgery. General anaesthesia combined with paravertebral versus serratus-intercostal block. Rev Esp Anestesiol Reanim. 2016;63(10):564-71. DOI: 10.1016/j. redar.2016.03.006.

6. Ahiskalioglu EO, Ahiskalioglu A, Aydin P, Yayik AM, Temiz A. Effects of single-dose preemptive intravenous ibuprofen on postoperative opioid consumption and acute pain after laparoscopic cholecystectomy. Medicine (Baltimore). 2017;96(8):e6200. DOI: 10.1097/ MD.0000000000006200.

7. Gordon DB, de Leon-Casasola OA, Wu CL, Sluka KA, Brennan TJ, Chou R. Research gaps in practice guidelines for acute postoperative pain management in adults findings from a review of the evidence for an American Pain Society clinical practice guideline. J Pain. 2016;17:158-66. DOI: 10.1016/j.jpain.2015.10.023.

8. Simpson JC, Bao X, Agarwala A. Pain management in Enhanced Recovery after Surgery (ERAS) protocols. Clin Colon Rectal Surg. 2019;32:121-8. DOl: 10.1055/s-0038-1676477.

9. Gan TJ. Poorly controlled postoperative pain: prevalence, consequences, and prevention. J Pain Res. 2017;10:228798. DOl: 10.2147/JPR.S144066.

10. Barazanchi AWH, MacFater WS, Rahiri JL, Tutone S, Hill $A G$, Joshi GP. Evidence-based management of pain after laparoscopic cholecystectomy: a PROSPECT review update. $\mathrm{Br} J$ Anaesth. 2018;121:787-803. DOI: 10.1016/j. bja.2018.06.023.

11. Teixeira UF, Goldoni MB, Machry MC, Ceccon PN, Fontes PR, Waechter FL. Ambulatory laparoscopic cholecystectomy is safe and cost-effective: a Brazilian single center experience. Arq Gastroenterol. 2016;53(2):103-7. DOI: 10.1590/ S0004-28032016000200010.

12. Tebala GD, Belvedere A, Keane S, Khan AQ, Osman A. Daycase laparoscopic cholecystectomy: analysis of the factors allowing early discharge. Updates Surg. 2017;69(4):461-9. DOI: 10.1007/s13304-017-0433-0.

13. Meissner W, Huygen F, Neugebauer EAM, Osterbrink J, Benhamou D, Betteridge N, et al. Management of acute pain in the postoperative setting: the importance of quality indicators. Curr Med Res Opin. 2018;34(1):187-96. DOI: 10.1080/03007995.2017.1391081.

14. López-Torres López J, Piedracoba Cadahía D, Alcántara Noalles MJ, Simó Cortés T, Argente Navarro P. Factores perioperatorios que contribuyen a la aparición de dolor o náuseas y vómitos postoperatorios en cirugía laparoscópica ambulatoria. Rev Esp Anestesiol Reanim. 2019;66(4):18998. DOI: 10.1016/j.redar.2018.11.007

15. Sangesland A, Støren C, Vaegter HB. Are preoperative experimental pain assessments correlated with clinical pain outcomes after surgery? A systematic review. Scand J Pain. 2017;15:44-52. DOl: 10.1016/j.sjpain.2016.12.002.

16. Zaballos M, Reyes A. Response to the letter to the editor "Anesthesia technique and quality of recovery after laparoscopic cholecystectomy: Case closed?» Rev Esp Anestesiol Reanim. 2018;65(7):417-8.

17. Le V, Kurnutala L, Schiano di Cola J, Ahmed K, Yarmush J, Eloy JD, et al. Premedication with intravenous ibuprofen improves recovery characteristics and stress response in adults undergoing laparoscopic cholecystectomy: a randomized controlled trial. Pain Med. 2016;17(6):1163-73. DOI: 10.1093/pm/pnv113.

18. de'Angelis N, Petrucciani N, Giannandrea G, Brunetti F. The protocol of low-impact laparoscopic cholecystectomy: the combination of mini-laparoscopy and low-pressure pneumoperitoneum. Updates Surg. 2018;70(4):553-6. DOI: 10.1007/s13304-018-0591-8.

19. Tharanon C, Khampitak K. The effect of peritoneal gas drain on postoperative pain in benign gynecologic laparoscopic surgery: A double-blinded randomized controlled trial. Int $\mathrm{J}$ Womens Health. 2016;10:373-9. DOI: 10.2147/IJWH. S109568.

20. Nadeem F, Khan MR, Naz FU. Comparison of mean pain scores for the patients with sub hepatic drainage to those without it after elective uncomplicated laparoscopic cholecystectomy. Pak J Med Sci. 2019;35(1):226-9. DOI: 10.12669/pjms.35.1.224.

21. Fernández Martín MT, López Álvarez S, Pérez Herrero MA. Serratus-intercostal interfascial block as an opioidsaving strategy in supra-umbilical open surgery. Rev Esp Anestesiol Reanim. 2018;65(8):456-60. DOI: 10.1016/j. redar.2018.03.007.

22. Lezana Pérez MÁ, Carreño Villarreal G, Lora Cumplido P, Alvarez Obregón R. Comparative study of ambulatory laparoscopic cholecystectomy versus management of laparoscopic cholecystectomy with conventional hospital stay. Cir Esp. 2013;91(7):424-31. DOI: 10.1016/j. ciresp.2012.10.010.

23. Sparber LS, Lau CS, Vialet TS, Chamberlain RS. Preoperative intravenous ibuprofen does not influence postoperative narcotic use in patients undergoing elective hernia repair: a randomized, double-bind, placebo controlled prospective trial. J Pain Res 2017;10:1555-60. DOI: 10.2147/JPR. S132836. 
24. Samuels DJ, Camporesi EM. Do not opiate! Anesthesiologists must be prepared to care for patients who sign a "non opioid directive". Anesth Analg. 2018;127(1):318-9. DOI: 10.1213/ANE.0000000000003403.

25. Moore RA, Derry S, Aldington D, Wiffen PJ. Single dose oral analgesics for acute postoperative pain in adults-an overview of Cochrane reviews. Cochrane Database Syst Rev. 2015;(9):CD008659. DOI: 10.1002/14651858. CDO08659.pub3.

26. Gregory J, McGowan L. An examination of the prevalence of acute pain for hospitalised adult patients: a systematic review. J Clin Nurs. 2016;25(5-6):583-98. DOI: 10.1111/ jocn. 13094.

27. Stessel B, Boon M, Pelckmans C, Joosten EA, Ory JP, Wyckmans W, et al. Metamizole vs. ibuprofen at home after day case surgery: A double blind randomised controlled noninferiority trial. Eur J Anaesthesiol. 2019;36(5):351-9. DOI: 10.1097/EJA.0000000000000972.

28. Lin YC, Chen CY, Liao YM, Lin PC, Chang CC. Pain relief by parecoxib for laparoscopic cholecystectomy: a meta-analysis of randomized controlled trials. Asian J Anesthesiol. 2018;56(3):92-114. DOI: 10.6859/ aja.201809_56(3).0004.

29. Kamali A, Ashrafi TH, Rakei S, Noori G, Norouzi A. A comparative study on the prophylactic effects of paracetamol and dexmedetomidine for controlling hemodynamics during surgery and postoperative pain in patients with laparoscopic cholecystectomy. Medicine (Baltimore). 2018;97(51):e13330. DOI: 10.1097/MD.0000000000013330.
30. Chilkoti GT, Kumar M, Mohta M, Saxena AK, Sharma N, Singh J. Comparison of postoperative analgesic efficacy of low-dose bolus intravenous dexmedetomidine and intraperitoneal dexmedetomidine with bupivacaine in patients undergoing laparoscopic cholecystectomy: A randomised, controlled trial. Indian J Anaesth. 2019;63(2):106-13. DOI: 10.4103/ija.IJA_440_18.

31. Gago Martínez A, Escontrela Rodriguez B, Planas Roca A, Martínez Ruiz A. Intravenous ibuprofen for treatment of postoperative pain: a multicenter, double blind, placebo-controlled, randomized clinical trial. PLoS One. 2016;11(5):e0154004. DOI: 10.1371/journal.pone.0154004. DOI: 10.1371/journal.pone. 0154004

32. Schwenkglenks M, Gerbershagen HJ, Taylor RS, Pogatzki-Zahn E, Komann M, Rothaug J, et al. Correlates of satisfaction with pain treatment in the acute postoperative period: results from the international Pain Out registry. Pain. 2014;155(7):140111. DOl: 10.1016/j.pain.2014.04.021.

33. Piirainen A, Kokki H, Immonen S, Eskelinen M, Häkkinen MR, Hautajärvi $\mathrm{H}$, et al. A dose-finding study of dexketoprofen in patients undergoing laparoscopic cholecystectomy: A randomized clinical trial on effects on the analgesic concentration of oxycodone. Drugs. 2015;15(4):319-28. D0I: 10.1007/ s40268-015-0107-7.

34. Gerbershagen HJ, Aduckathil S, van Wijck AJ, Peelen LM, Kalkman CJ, Meissner W. Pain intensity on the first day after surgery: a prospective cohort study comparing 179 surgical procedures. Anesthesiology. 2013;118(4):934-44. DOI: 10.1097/ALN.0b013e31828866b3. 